\title{
Global quantitative biology can illuminate ontological connections between diseases
}

\author{
Guanyu Wang* \\ Department of Biology, Southern University of Science and Technology, Shenzhen 518055, China \\ * Correspondence: wanggy@sustc.edu.cn
}

Received December 8, 2016; Revised January 23, 2017; Accepted January 24, 2017

\begin{abstract}
Owing to its interdisciplinary nature, quantitative biology is playing ever-increasing roles in biological researches. To make quantitative biology even more powerful, it is important to develop a holistic perspective by integrating information from multiple biological levels and by considering related biocomplexity simultaneously. Using complex diseases as an example, $I$ show in this paper how their ontological connections can be revealed by considering the diseases on a common ground. The obtained insights may be useful to the prediction and treatment of the diseases. Although the example involves only with cancer and diabetes, the approaches are applicable to the study of other diseases, or even to other biological problems.
\end{abstract}

Keywords: quantitative biology; disease modeling; systems biology; nonlinear dynamics

\section{INTRODUCTION}

The 21 st century has witnessed flourish of quantitative biology, which includes two broad and related areas. The first is bioinformatics and computational biology, which focuses on dealing with information technologies and computational methodologies that can efficiently and accurately manipulate omics data and transform molecular information into biological knowledge [1]. The other is systems and synthetic biology, which focuses on complex interactions in biological systems and the emergent functional properties, and on the design and construction of new biological functions and systems. These rapid developments notwithstanding, quantitative biology is often regarded to be secondary to the traditional biology. Biological researches generally start from a working hypothesis. To test the hypothesis, one performs experiments, from which data are generated. There are generally two approaches to process the data. The first is the model driven approach, which relies on a mathematical model of the underlying biological system. The second is the data driven approach, whereby patterns in the data can be discovered by exploiting generic statistical and/or dynamical properties, without a specific model of the underlying system [2]. Because most collaborations between biologists and quantitative scientists do follow the aforementioned way, many get the erroneous impression that quantitative biology is non-essential and mathematical models are only tools to elaborate empirically-derived biological patterns but not to independently make substantial new advances [3].

Owing to its interdisciplinary nature, quantitative biology actually can achieve a holistic grasp of biological systems through integrating information spanning multiple levels from molecules to the organism. In comparison, traditional biological studies usually follow a reductionist approach, dividing the complex cell into many individual parts, studying each part in great detail, and then trying to understand the whole cell by integrating partial information. This reductionist approach has been successful, with the help from technological advancements that allow us to penetrate into the subcellular world and to quantify its fine details. However, unlike a linear system in which the whole is always the sum of its constituents, biological systems are highly nonlinear; and simple accumulation of local data does not automatically lead to the emergence of systems-level properties [4,5]. To achieve a quantum leap of our understanding of life, interdisciplinary sciences such as quantitative biology are desperately needed.

To make quantitative modeling more powerful, the key is to look at many problems at once and to obtain a global quantitative characterization of the biological system 
under study. To that end, it is imperative to gather information from multiple biological levels (evolutionary scale, organismal, cellular and molecular levels) so that one develops the necessary vision to ponder fundamental aspects of biology and to discover emerging properties from mathematical modeling and analysis. In the following, I will show how the philosophy of global quantitative biology helps to study complex diseases. Instead of studying one specific disease, the conceptual framework requires the study of multiple related diseases together, from which novel insights can be gained including ontological connections between the diseases.

\section{ANALYSIS OF DIFFERENT DISEASES ON A COMMON GROUND}

One major health care problem of our time is the simultaneous epidemics of cancer and diabetes, which are escalating in recent years [6,7]. In the US, about two thirds of the population is overweight; about one third of children born after year 2000 would develop diabetes; about one third of women and half men would develop cancer during their lifetime [8]. These epidemiological data imply that cancer and diabetes have intricate connections. Many clinical studies also arrived at the same conclusion. Patients diagnosed with metabolic diseases showed increased incidence and aggressiveness of cancer development [9,10]. Conversely, diabetics treated with metformin to lower insulin levels have reduced chances of cancer in comparison to untreated individuals $[11,12]$. However, these data basically just say that the two diseases can promote each other, which are not essential connections.

\section{Ontological connection between the diseases}

Ontological connection refers to how the nature of beings is related. Because a single disease is already complex, it seems difficult to examine multiple diseases on a common ground and even more difficult to seek their ontological connections. Fortunately, most complexities of a disease are only symptoms, namely non-essential body reactions; they are not defining features of the disease and are less relevant when considering ontological connections. Dating back from the onset of symptoms, there was a long period of clinical latency, during which the disease state is only slightly different from the normality. In the following, we still use "disease" to refer to its existence during the latency phase, although 'pre-disease' may be more accurate. Therefore, a disease is intimately connected with the normality: their difference is small but is gradually amplified. Since the normality can be disturbed in different ways, one might as well identify all the patterns of deviation around the normality. Each pattern represents a family of disturbances that are qualitatively the same; and may correspond to a specific disease. In this way, one can include multiple diseases into the same global picture, in which the diseases are qualitatively different but interconnected entities that bifurcate from the normality (Figure 1A). Such a holistic perspective could facilitate a deeper understanding of complex diseases and contribute to their prevention and treatments.

Figure 1A is just a sketch of disease evolution. How to map it to a biologically meaningful one? One needs to consider these diseases within the same conceptual framework, including a candidate biological system that underlies all these diseases. By mathematical modeling on the biological system, one may find characteristic representations (e.g., geometric objects) of these diseases. By presenting these geometric objects in the same global picture, their ontological connections may become intuitive. The scheme is generally practical, because one needs only to consider the latency phase, during which the deviation of diseases from the normality is quite small. In this event, infinitesimal methods of mathematical analysis can be used to study the early evolution of the diseases.

It should be emphasized that the intricacy of the discovered ontological connections depends on the complexity of the underlying biological system. By studying a relatively small system, one has better chance to uncover the ontological connections with reasonable computational costs. Although the uncovered connections are likely to be limited, they may provide hitherto unimagined insights and constitute a good starting point for future researches.

\section{Molecular network underlying cancer and diabetes}

In the cell, it is the complex interaction network between biomolecules that provides functional output. By examining major signaling pathways, I found that many of them are relevant to cancer and diabetes, with the PI3K/ Akt/mTOR pathway especially important. This pathway is a cellular pivot responding to extracellular stimuli such as insulin, insulin-like growth factor (IGF), epidermal growth factor (EGF), and fibroblast growth factor (FGF). It carries out a large spectrum of biological functions including cell survival, growth, migration, proliferation, polarity, and metabolism (lipid and glucose); cell cycle progression; muscle and cardiomyocyte contractility; angiogenesis; and self-renewal of stem cells [13-15]. Unsurprisingly, deregulation of the pathway is implicated in many diseases including cancer and diabetes [16]. Among the signaling molecules of the pathway, Akt (also known as the protein kinase B) is the central regulator and its level of phosphorylation is an indicator of the pathway activity. I therefore used the response curve $A(I)$ to define the pathway functionality [17], where $A$ represents the 

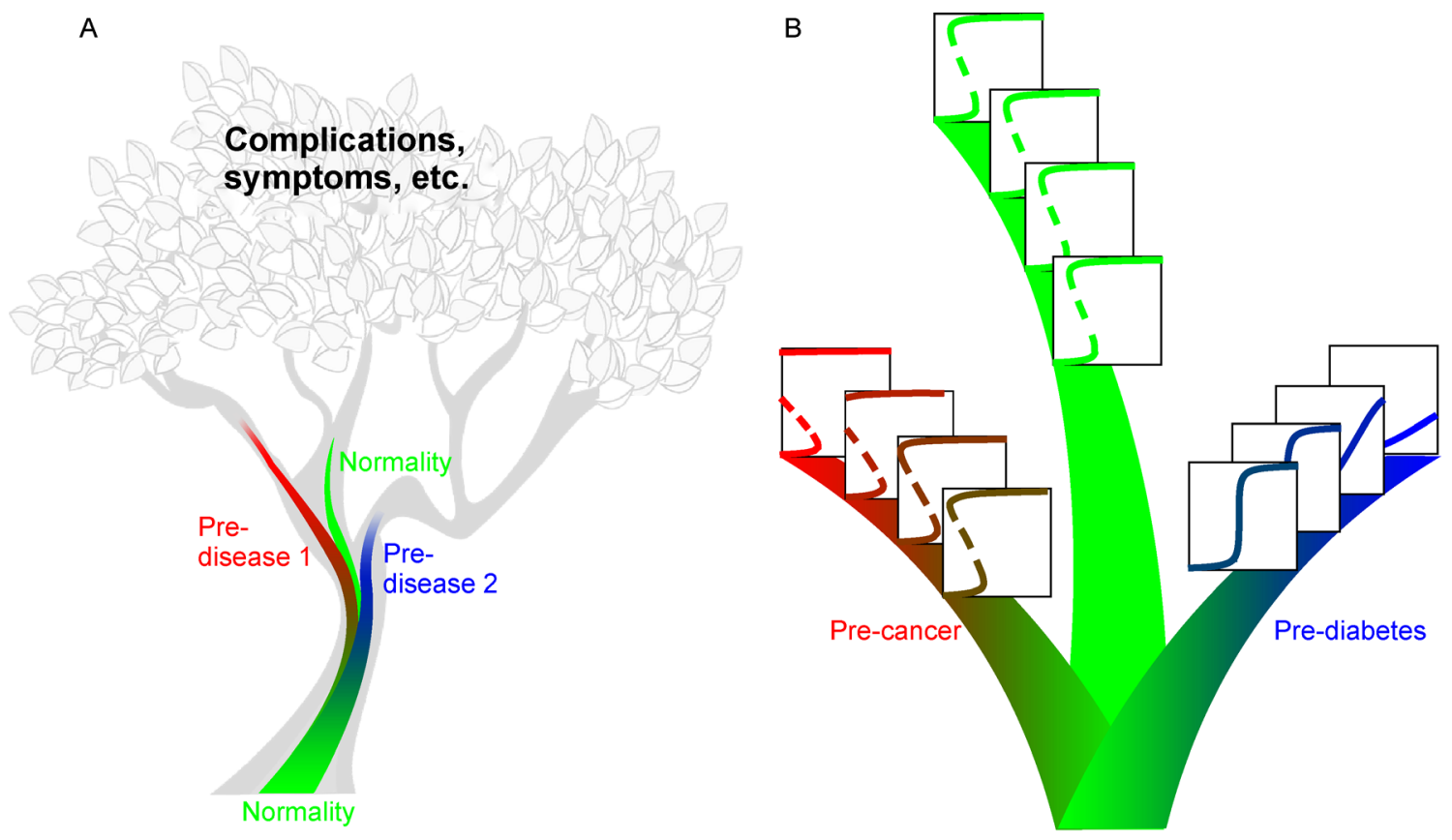

Figure 1. Evolution of diseases. (A) A sketch. (B) A hypothetical, biologically meaningful illustration.

percentage of the phosphorylated Akt over the total Akt; I represents the stimulus concentration. In the following, the shape of $A(I)$ is used to characterize the health state.

\section{Mathematical analysis of the molecular network}

Although a disease may come into being by gradual deviating from the normality, it will become qualitatively different from the normality sooner or later. In terms of the response curve $A(I)$, this means that its shape deviates slowly from normal throughout the time. Initially the change is only quantitative, but at some time point a transition occurs, after which $A(I)$ becomes qualitatively different. This qualitative transition can be mathematically characterized by catastrophe theory, or singularity theory for some authors [17-19]. The boundary between qualitatively different entities is called singularity. Dynamics around singularities is interesting and has been exploited to study important biological problems $[2,20,21]$.

Being mathematically special, singularities are much easier to determine (numerical computations can be simplified or even avoided by applying analytical techniques). Once the boundaries (each consisting of many singularities) are determined, the landscape of responses has already been illuminated. To understand this argument, one may use an analogy with drawing an object. By tracing out the boundary between the object and air, the object has already been drawn. One needs not to fill in every point of the object.
$[17$

The steady state of the pathway can be described by

$$
G(A, I, K, a)=0,
$$

where $K$ and $\alpha$ are two distinguished parameters in the system. Because $A, I, K$, and $\alpha$ are constrained by Equation (1), the shape of $A(I)$ changes when $K$ or $\alpha$ changes. On the plane spanned by $K$ and $\alpha$, each point corresponds to a shape of $A(I)$. The plane can thus be divided into different regions, each consisting of points whose corresponding response curves are of the same kind. The boundary between two regions consists of those points whose corresponding $A(I)$ has "critical shape". These boundary points are known as nonpersistent points [19]. There are four kinds of nonpersistent points: hysteresis point, isola center, simple bifurcation point, and double limit points [19]. They are described respectively in the first, second, third, and fourth rows of Figure 2. In the first row, for example, the middle graph presents $A(I)$ with the corresponding $(K, \alpha)$ being the hysteresis point, while the left and right graphs present $A(I)$ when $(K, \alpha)$ are perturbed away from the hysteresis point.

The four kinds of nonpersistant points all satisfy

$$
\begin{gathered}
G=0, \\
G_{A}=0,
\end{gathered}
$$

where Equation (2) is the same as Equation (1); and $G_{A}$ in Equation (3) denote $\partial G / \partial A$. Similarly, $G_{A A}$ shall be used to denote $\partial^{2} G / \partial A^{2}$, etc. To distinguish these nonper- 

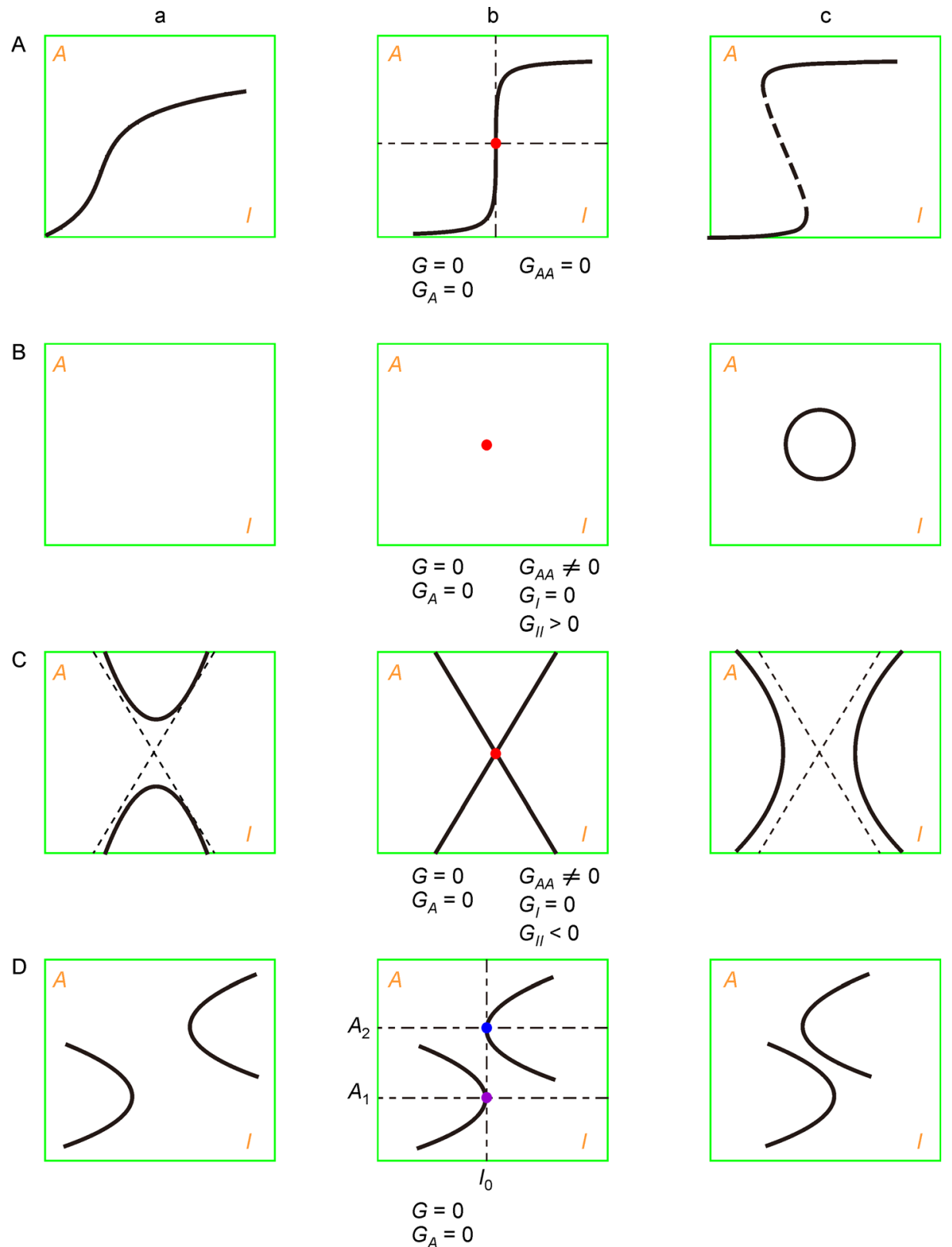

Figure 2. The four types of nonpersistent points (the middle column) and their perturbations (the left and right columns).

(A) Hysteresis point. (B) Isola center. (C) Simple bifurcation. (D) Double limit points.

sistant points, additional conditions are required. Their normal form conditions are written with red color in Figure 2. The "double limit points", for example, correspond to the two solutions to Equations (2) and (3). They are indicated by the blue and purple dots in Figure 2D-b. In contrast, all the other three nonpersistent points correspond to the unique solution to their respective normal form equations.

The present mathematical model has one hysteresis point solution but no solutions of isola center, simple bifurcation point, and double limit points [17]. As the parameters change, the hysteresis point changes accordingly, tracing out the blue curve in Figure 3. One sees that it is indeed a boundary dividing the parameter plane into two regions. In the region below the blue curve, any point's corresponding response curve is a monotonically increasing function. In the region above the blue curve, any point's corresponding response to the stimulus manifests bistability.

The area above the blue curve can be further divided. A bistable response has two thresholds $I_{\text {on }}$ and $I_{\text {off }}$. The sign of $I_{\mathrm{off}}$ is of great biological significance. When $I_{\mathrm{off}}<0$, 


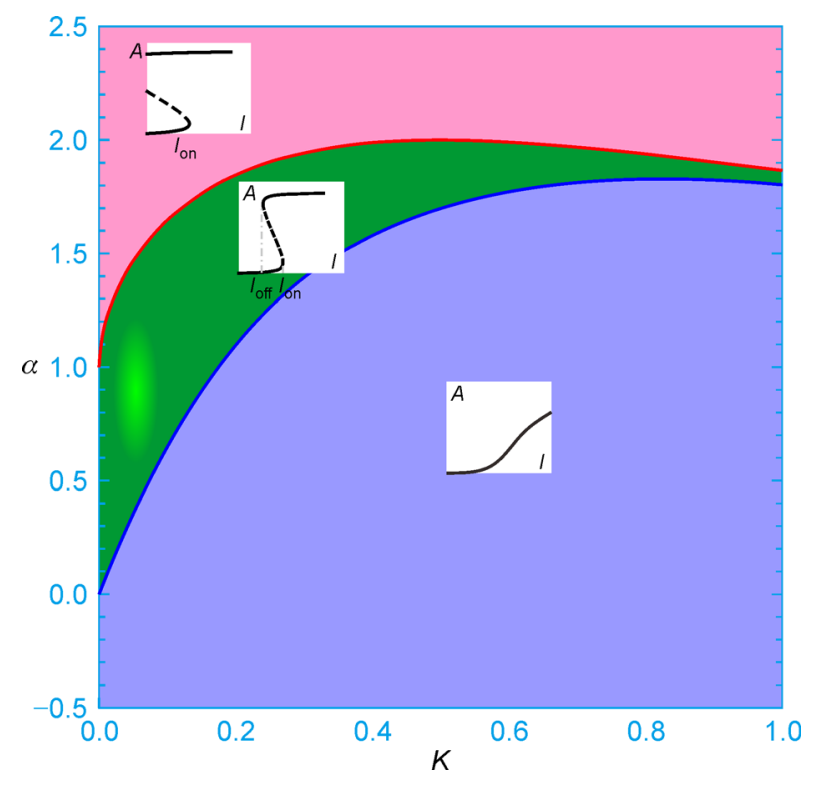

Figure 3. A phase diagram of physiologic and pathologic phenotypes arising from the PI3K/Akt/mTOR pathway. The graph $A(I)$ is the response curve of the pathway. $K$ and $\alpha$ are two distinguished parameters of the system. As the two parameters change, the shape of $A(I)$ changes. The phase diagram reveals three qualitatively different response curves, which from the top down are the irreversible switch, the toggle switch, and the monotone type.

the "jump-down" part of the response curve is cut off by the vertical axis, which causes constitutive activation of Akt. That is, once Akt is activated, it will remain activated, even after the stimulus is completely gone. This subtype is thus called irreversible switch. When $I_{\text {off }}>0$, both activation and deactivation of Akt are under the control of realistic insulin concentrations; and this subtype is called toggle switch. In Figure 3, the red curve corresponds to the critical condition $I_{\text {off }}=0$, which separates the region with $I_{\text {off }}<0$ from the region with $I_{\text {off }}>0$.

\section{Phase diagram of the diseases}

Mathematical analysis has revealed that the PI3K/Akt/ mTOR pathway has only three kinds of steady-state response. They are presented in Figure 3, which from the top down are the irreversible switch, the toggle switch, and the monotone type. Because no other steady-state responses exist [17], it is reasonable to relate the three kinds of response to physiologic/pathologic phenotypes of the pathway.

Cancer. The irreversible switch shown in Figure 3 may correspond to the cancer phenotype. Suppose initially the concentration of the growth factor (insulin, insulin-like growth factor, epidermal growth factor, etc. $)$ is zero $(I=0)$ and Akt is not activated $(A=0)$. As $I$ increases over the threshold $I_{\mathrm{on}}$, Akt is fully activated $(A \approx 1)$. Remarkably, this activation becomes permanent. To deactivate Akt, the growth factor concentration has to be negative (to make $I$ even smaller than the negative $I_{\text {off }}$ ), which is impossible. In this event, a transient stimulus gives rise to constitutively active Akt. That is, a single pulse of the growth factor can trigger the permanent activation of Akt, as long as the peak of the pulse is over $I_{\mathrm{on}}$. This activation pertains even if future stimulations will never arrive. It is well known that Akt is an oncoprotein that promotes carcinogenesis from many aspects. The constitutive activation of Akt promotes massive and continuous glucose uptake, which is necessary for cancer metabolism and uncontrolled cell proliferation [22]. Akt activation also promotes cell survival through coupling with other pathways such as NFKB [23]. Even if the local environment has very low glucose concentration, the cell may still manage to survive. It also promotes metastasis through decreasing transcription of the transmembrane protein E-cadherin that forms adhesions between adjacent cells, thereby permitting their detachment and migration to a new environment that is richer in nutrients [24]. Therefore, the pink region in Figure 3 should be cancer-prone, because all the irreversible switches locate in the region. It is possible that the entire cancer-prone region is even larger, encroaching the green region.

Diabetes. The monotone type shown in the light blue region of Figure 3 may correspond to a stifled Akt activation of myocytes in response to insulin stimulus. That is, Akt activity is low under the stimulation of normal postprandial insulin. Because Akt activation drives the myocyte's glucose uptake, the low Akt activity impairs glucose disposal. To bring plasma glucose concentration under control, the pancreas has to produce much more insulin so that Akt can be fully activated. The overproduction of insulin would gradually exhaust the pancreas (e.g., the destruction of islet cells) and lead to its failure. Finally, the pancreas can no longer secrete the amount of insulin it did previously. The weakened insulin secretion, together with the aberrant response curve, leads to the full-blown diabetes: glucose cannot be utilized by the majority of tissue cells and has to remain in the blood (hyperglycemia). All in all, the deformation of bistability into the monotone type increases the risk of type 2 diabetes. Therefore, the light blue region in Figure 3 should be diabetes-prone.

Normality. Because the upper and lower regions manifest anormalities, the normal response should be toggle switch, for the majority of cell types. Taking toggle switch as a normal mode of response is quite reasonable. It is well known that bistability has many fine properties that are useful for cellular functioning [25]. For example, 
it produces a "memory" of a transient stimulus, which is important for cell differentiation and cell cycle progression [26]; it helps to achieve biological rhythms with widely tunable frequency and near-constant amplitude [27]. Notably, I find that bistability emerges as an indispensable control mechanism to achieve optimal homeostasis, including glucose homeostasis mediated by the PI3K/Akt/mTOR pathway [28]. Bistability appears to be the only solution to a dilemma in glucose homeostasis: high insulin efficiency is required to confer rapidness in plasma glucose clearance, whereas an insulin sparing state is required to guarantee the brain's safety during fasting. Therefore, normal cells, especially normal myocytes, may use toggle switch to respond to insulin. That is, points corresponding to the normal response should locate within the middle green region, for example the bright green region.

\section{Bifurcation of the diseases}

The obtained phase diagram reveals all the kinds of responses constrained by the $\mathrm{PI} 3 \mathrm{~K} / \mathrm{Akt} / \mathrm{mTOR}$ pathway. It also suggests the way anormalities could arise through gradual departure from normality (Figure 1B). Dysregulations of the PI3K/Akt/mTOR pathway can be caused by many factors including genetic, molecular, and environmental ones. The accumulation of these abberations drives the deformation of the response curve and finally leads to various diseases. The left trajectory in Figure 1B may represent one typical scenario. A subject may have a cell with ever increasing genomic instability, which causes the $I_{\text {off }}$ value of the cell's response curve reduces gradually until finally turns into negative, forming an irreversible switch. The cell consequentially becomes transformed due to the constitutive activation of Akt. Another scenario may be represented by the right trajectory in Figure 1B. A subject's myocytes and adipocytes may become more and more insulin resistant, caused by the flattening of their response curves. The subject may finally develop diabetes as a consequence. Importantly, we now have a biologically meaningful disease evolution (Figure 1B) mapping to the sketch evolution (Figure 1A).

\section{Yin-Yang polarity}

Figure 3 reveals an interesting polarity: cancer and diabetes phenotypes are opposite to each other, flanking the normality phenotype. The polarity arises from the cell's response to growth factors such as insulin. Diabetes is characterized by insulin insensitivity, whereas cancers are characterized by insulin hypersensitivity. Normality corresponds to medium insulin response. Interestingly, the medium response is not simply averaging maximal and minimal Akt activation, but a controllable switching between maximal and minimal. It is the concept of ontological connection that leads to the global analysis of the PI3K/Akt/mTOR pathway and thus Figure 3, which in turn leads to intuitive insights into disease evolution (Figure 1B).

Ontological connections between cancer and diabetes emphasize the common grand of the two polar opposites, which is reminiscent of the concept of Yin-Yang (literally "shadow-light") in traditional Chinese philosophy. This concept also emphasizes the mutual whole, on the basis of which polar opposites or seemingly contrary forces are interconnected or even interconverted. This mutual whole, as well as the principle governing its interconversion, is known as Tao in Chinese philosophy: "everything carries Yin yet embraces Yang, with a mellowing energy for harmony" [29].

\section{CONCLUSION}

Quantitative design and analysis are playing everincreasing roles in biological researches [30-32]. With a holistic perspective, quantitative biology can provide hitherto unimagined insights into vital biological problems such as diseases, their simultaneous epidemics, and their ontological connections. Because cancer and diabetes correspond to opposite deformations to the normal bistability, they are yet another manifestation of Yin-Yang polarity, besides natural dualities such as hot and cold, light and dark, etc. The conclusion is resonant with the holistic theory characteristic of traditional Chinese medicine, whose studies also require global nonlinear analysis instead of traditional reductionist approaches. Recently, a network targeted approach has been introduced to illustrate the complex interactions among the biological systems, drugs, and complex diseases from a network perspective, which shed light on approaches to exploit traditional Chinese medicine $[33,34]$.

Because cancer and diabetes are contrary to each other, one may conclude that a cancer patient is difficult to contract diabetes, and vice versa. This conclusion is of cause wrong. A multicellular organism has many cells. And it is quite natural that one cell adopts irreversible switch as its response curve, another cell adopts monotone type, and yet another cell adopts the normal toggle switch. For example, a diabetes patient's myocytes may all become severely insulin resistant; and this does not hinder a hepatocyte to become cancerous. In fact, this may even be advantageous to the transformation of the hepatocyte, because the diabetes patient often has high glucose concentration in the blood (hyperglycemia). Because a tumor utilizes far greater amounts of glucose than normal tissues, muscles' insulin resistance should be 
beneficial to the tumor growth. This possibility may explain the simultaneous escalation of the epidemics of cancer and diabetes. The two diseases are the two sides of the same coin. This mutual reinforce between opposing entities is also a character of Yin-Yang.

With the obtained insights, a natural step forward would be performing experiments to map theoretical response curves to actual cellular responses. One can measure the level of Akt phosphorylation at different concentrations of a growth factor. To measure the reverse direction response, one can first prime the cells at a high growth factor concentration and then repeat the same procedure of the forward direction. To remove the effects of heterogeneity, one can use single cell fluorescence imaging. Fluorescent indicators have been developed for key molecules in the PI3K/Akt/mTOR pathway, including AKT, GLUT4, and PI3K [35,36]. It would be important to examine cells as diverse as possible. For example, one can examine different cells of the same subject to determine heterogeneity across cell types and heterogeneity within the same cell type. One can also examine the same type of cells undergoing various pathologic changes to determine the corresponding deformations of the response curve. Another interesting direction is to study stochastic fluctuations in molecular concentrations in the $\mathrm{PI} 3 \mathrm{~K} / \mathrm{Akt} / \mathrm{mTOR}$ pathway. One merit of bistability is to confer robust switching in the face of fluctuations, which is important to the functional outputs of the PI3K/Akt/mTOR pathway. To avoid hyperglycemia, for example, a stable glucose disposal is required.

Although we have used cancer and diabetes as an example, the approach introduced in the present paper may be used to uncover ontological connections of other categories of diseases, for example the connection between Parkinson's disease and Alzheimer's disease.

\section{COMPLIANCE WITH ETHICS GUIDELINES}

The author Guanyu Wang declare that he has no conflict of interests. All the data sets the author used are from public repositories.

This article does not contain any studies with human or animal subjects performed by the author.

\section{REFERENCES}

1. Guan, L., Yang, Q., Gu, M., Chen, L. and Zhang, X. (2014) Exon expression qtl (eeqtl) analysis highlights distant genomic variations associated with splicing regulation. Quant. Biol., 2, 71-79

2. Chen, L., Liu, R., Liu, Z.-P., Li, M. and Aihara, K. (2012) Detecting early-warning signals for sudden deterioration of complex diseases by dynamical network biomarkers. Sci. Rep., 2, 342

3. Servedio, M. R., Brandvain, Y., Dhole, S., Fitzpatrick, C. L., Goldberg, E. E., Stern, C. A., Van Cleve, J. \& Yeh, D. J. (2014) Not just a theory - the utility of mathematical models in evolutionary biology. PLoS. Biol., 12, e1002017

4. Nagel, E. and Hawkins, D. (1961) The structure of science. Am. J. Phys., 29, 716

5. Bruggeman, F. J., Westerhoff, H. V. and Boogerd, F. C. (2002) Biocomplexity: a pluralist research strategy is necessary for a mechanistic explanation of the "live" state. Philos. Psychol., 15, 411440

6. Bergman, M. (2013) Pathophysiology of prediabetes and treatment implications for the prevention of type 2 diabetes mellitus. Endocrine, $43,504-513$

7. Smyth, S. and Heron, A. (2006) Diabetes and obesity: the twin epidemics. Nat. Med., 12, 75-80

8. Mukherjee, S. (2010) The Emperor of All Maladies: a Biography of Cancer. New York: Scribner

9. Giovannucci, E., Harlan, D. M., Archer, M. C., Bergenstal, R. M., Gapstur, S. M., Habel, L. A., Pollak, M., Regensteiner, J. G. and Yee, D. (2010) Diabetes and cancer: a consensus report. CA Cancer J. Clin., 60, 207-221

10. Pischon, T., Nöthlings, U. and Boeing, H. (2008) Obesity and cancer. Proc. Nutr. Soc., 67, 128-145

11. Hsu, I. R., Kim, S. P., Kabir, M. and Bergman, R. N. (2007) Metabolic syndrome, hyperinsulinemia, and cancer. Am. J. Clin. Nutr., 86, s867s871

12. Larsson, S. C., Mantzoros, C. S. and Wolk, A. (2007) Diabetes mellitus and risk of breast cancer: a meta-analysis. Int. J. Cancer, 121, 856862

13. Engelman, J. A., Luo, J. and Cantley, L. C. (2006) The evolution of phosphatidylinositol 3-kinases as regulators of growth and metabolism. Nat. Rev. Genet., 7, 606-619

14. Liao, Y. and Hung, M.-C. (2010) Physiological regulation of Akt activity and stability. Am. J. Transl. Res., 2, 19-42

15. Li, T. \& Wang, G. (2014) Computer-aided targeting of the PI3K/Akt/ mTOR pathway: toxicity reduction and therapeutic opportunities. Int. J. Mol. Sci., 15, 18856-18891

16. Zoncu, R., Efeyan, A. and Sabatini, D. M. (2011) mTOR: from growth signal integration to cancer, diabetes and ageing. Nat. Rev. Mol. Cell Biol., 12, 21-35

17. Wang, G. (2010) Singularity analysis of the AKT signaling pathway reveals connections between cancer and metabolic diseases. Phys. Biol. 7, 046015

18. Arnold, V. (1986) Catastrophe Theory. Berlin: Springer-Verlag

19. Golubitsky, M. and Schaeffer, D. G. (1985) Singularities and Groups in Bifurcation Theory. New York: Springer-Verlag

20. Liu, R., Aihara, K. and Chen, L. (2013) Dynamical network biomarkers for identifying critical transitions and their driving networks of biologic processes. Quant. Biol., 1, 105-114

21. Liu, R., Chen, P., Aihara, K. \& Chen, L. (2015) Identifying earlywarning signals of critical transitions with strong noise by dynamical network markers. Sci. Rep., 5, 17501

22. Hong, S. Y., Yu, F.-X., Luo, Y. and Hagen, T. (2016) Oncogenic activation of the PI3K/Akt pathway promotes cellular glucose uptake by downregulating the expression of thioredoxin-interacting protein. Cell. Signal., 28, 377-383

23. Zhu, X., Song, Y., Wu, C., Pan, C., Lu, P., Wang, M., Zheng, P., Huo, R., Zhang, C., Li, W. et al. (2016) Cyr61 participates in the pathogenesis of acute lymphoblastic leukemia by enhancing cellular survival via the Akt/NFB signaling pathway. Sci. Rep., 6, 34018 
24. Wang, H., Fan, L., Wei, J., Weng, Y., Zhou, L., Shi, Y., Zhou, W., Ma, D. \& Wang, C. (2012) Akt mediates metastasis-associated gene 1 (MTA1) regulating the expression of E-cadherin and promoting the invasiveness of prostate cancer cells. PloS One 7, e46888

25. Tyson, J. J., Albert, R., Goldbeter, A., Ruoff, P. and Sible, J. (2008) Biological switches and clocks. J. R. Soc. Interface, 5, S1-S8

26. Xiong, W. and Ferrell, J. E. Jr. (2003) A positive-feedback-based bistable "memory module" that governs a cell fate decision. Nature, 426, 460-465

27. Tsai, T. Y.-C., Choi, Y. S., Ma, W., Pomerening, J. R., Tang, C. and Ferrell, J. E. Jr. (2008) Robust, tunable biological oscillations from interlinked positive and negative feedback loops. Science, 321, 126129

28. Wang, G. (2012) Optimal homeostasis necessitates bistable control. J. R. Soc. Interface, 9, 2723-2734

29. Tzu, L. (1972) Tao De Ching, translated by Feng, G. and English, J. New York: Vintage (Original work published CA. 350-250 BC)

30. Meng, H. and Wang, Y. (2015) Cis-acting regulatory elements: from random screening to quantitative design. Quant. Biol., 3, 107-114

31. Zhou, T. and Liu, T. (2015) Quantitative analysis of gene expression systems. Quant. Biol., 3, 168-181

32. Cui, H., Li, Y. and Zhang, X. (2016) An overview of major metagenomic studies on human microbiomes in health and disease. Quant. Biol., 4, 192-206

33. Li, R., Chen, T. and Li, S. (2015) Network-based method to infer the contributions of proteins to the etiology of drug side effects. Quant. Biol., 3, 124-134

34. Li, S. (2016) Exploring traditional chinese medicine by a novel therapeutic concept of network target. Chin. J. Integr. Med., 22, 647652

35. Tengholm, A., Teruel, M. N. and Meyer, T. (2003) Single cell imaging of PI3K activity and glucose transporter insertion into the plasma membrane by dual color evanescent wave microscopy. Sci. STKE, 2003, pl4.

36. Sato, M. (2006) Imaging molecular events in single living cells. Anal. Bioanal. Chem., 386, 435-443 